\title{
Discharges to prison from medium secure psychiatric units in England and Wales
}

Michael Doyle, Jeremy Coid, Laura Archer-Power, Lindsay Dewa, Alice Hunter-Didrichsen, Rachel Stevenson, Verity Wainwright, Costas Kallis, Simone Ullrich and Jenny Shaw

\section{Background}

Early findings from a national study of discharges from 32 National Health Service medium secure units revealed that nearly twice as many patients than expected were discharged back to prison.

\section{Aims}

To compare the characteristics of those discharged back to prison with those discharged to the community, and consider the implications for ongoing care and risk.

\section{Method}

Prospective cohort follow-up design. All forensic patients discharged from 32 medium secure units across England and Wales over a 12-month period were identified.

Those discharged to prison were compared with those who were discharged to the community.

\section{Results}

Nearly half of the individuals discharged to prison were diagnosed with a serious mental illness and over a third with schizophrenia. They were a higher risk, more likely to have a personality disorder, more symptomatic and less motivated than those discharged to the community.

\section{Conclusions}

Findings suggest that alternative models of prison mental healthcare should be considered to reduce risks to the patient and the public.

\section{Declaration of interests}

\section{None.}

Forensic mental health services are commissioned to prevent further offending and provide care and treatment in secure conditions. Medium secure psychiatric units were developed in England and Wales following the Butler Report, ${ }^{1}$ which highlighted the need for step-down services from the then overcrowded high secure psychiatric hospitals. Medium secure units (MSUs) aim to rehabilitate mentally disordered offenders to community-based services and bed numbers have expanded since the late 1990 s to nearly $40000^{2,3}$ However, there is little evidence that frequency of discharge has increased and the mean length of stay has become progressively longer. More than a quarter of patients in high and medium secure services now stay 10 years or longer. ${ }^{4}$ Prolonged stay is not explained by objective indicators of future risk of violence. Poor treatment response and whether the patient is detained under a Section 41 restriction order of the Mental Health Act appear to be the key factors that delay discharge, ${ }^{5,6}$ together with inadequate provision of alternative options for patient transfer. However, if offender patients are admitted from prison when serving a custodial sentence instead of on a hospital order, they can be returned back to prison following treatment. (Section 47 of the Mental Health Act 1983 allows for a transfer direction of a sentenced prisoner to hospital. Those transferred to hospital on Section 47 can be subsequently placed on a notional Section 37 treatment order or returned to prison.) This in turn maintains throughput of patients through the diminishing number of those beds that are occupied by long-stay patients.

In $1997 / 1998,12.8 \%$ of patients in 34 MSUs were discharged back to prison. ${ }^{7}$ However, we observed that in 2010-2011 the proportion from 32 National Health Service (NHS) MSUs in England and Wales had risen to $20 \%$. The aims of this study were to identify the reasons for the increase, compare the characteristics of those discharged back to prison with those discharged to the community, and consider the implications for aftercare and public risk.

\section{Method}

A prospective cohort follow-up design was chosen for this study, similar to the approach taken in the MacArthur Violence Risk Assessment Study $^{8}$ and more recent similar UK studies. ${ }^{9,10}$ All forensic patients discharged from 32 NHS MSUs across England and Wales during the period 1 September 2010 to 31 August 2011 were identified and those discharged to prison (prison discharge group) were compared with those who were discharged to the community and non-forensic placements (community discharge group).

\section{Procedure}

The North West England Multi Site Research Ethics Committee approved the study and, to ensure a total sample of discharges was included, permission was sought and granted by the National Information Governance Board (NIGB) to conduct the study without patient consent under Section 251 of the NHS Act 2006. A link person was identified at each of the hospital sites and a notification system was set up so that the researchers were automatically informed when a patient was discharged. Baseline assessments were then conducted for those who had been discharged to prison or community settings. This was conducted by interviewing a member of staff who knew the patient well and a review of clinical and criminal records.

\section{Baseline assessment measures}

Demographic and diagnostic information were recorded at baseline for each patient and the measures of risk factors, protective factors and mental state were completed based on information from a collateral interview and file review. The following assessments were conducted. 
(a) The Historical, Clinical and Risk - 20 items (HCR-20), ${ }^{11}$ which is a broadband violence risk assessment used widely in research and clinical practice. It comprises ten historical factors, five clinical items that are meant to reflect current, dynamic (changeable) correlates of violence and five risk management items, which focus on future factors. The items are scored 0 if item not present, 1 if item partially present, and 2 if item definitely present. Scores range 0-40. In this study, version 3 of the HCR-20 (HCR-20V3) ${ }^{12}$ was used to compare the risk between the prison discharge and community discharge groups.

(b) The Structured Assessment of Protective Factors (SAPROF), ${ }^{13}$ which is a guideline developed to measure protective factors that mitigate future risk of violence. There are 17 items covering internal, motivational and external factors that have been found to protect against violence. Each item is rated on a three-point scale $(0,1$ or 2$)$ and scores range between 0 and 34 .

(c) The Medium Secure Recidivism Assessment Guide (MSRAG) ${ }^{14}$ which is a 12 -item actuarial violence risk assessment tool validated in two parts to predict acquisitive recidivism and serious offending recidivism, from patients discharged from an MSU into the community up to 6 years post-discharge. Each of the items has a simple three-point rating $(-1,0,+1)$ except for one binary rating $(-1,+1)$. The scores range from -1 to 22 .

(d) The Positive and Negative Syndrome Scale (PANSS), ${ }^{15}$ which is a 33-item rating instrument that evaluates the severity of positive and negative symptoms, general psychopathology of schizophrenia and aggression. All items are rated on a seven-point scale ( 1 , absence to 7 , extreme) and scores range from 33 to 231. This was completed following a review of records and an interview with the collateral informant based on patient behaviour and functioning 1 month prior to discharge. This collateral plus record review method of measuring mental state without patient interview was found to be successful in previous studies of this type. ${ }^{8}$

(e) The Severity of Index Offence scale,${ }^{16}$ which is an eight-point scale $(0-7)$ that was designed specifically to measure the severity of index offence in detained male psychiatric patients in the USA. The scores range from 0 , 'no offence' to 7 'loss of life with extreme violence'.

\section{Participants}

Participants were identified as having been discharged from the MSU to one of four locations - a high secure unit (HSU), a low secure unit (LSU), prison or the community. All discharge locations other than to an HSU, LSU and prison were to non-secure and community settings. The definition of 'community' here encompasses various open types of accommodation, including independent tenancies, supported accommodation, hostels, open rehabilitation wards and open units.

\section{Data analysis}

Data were analysed using SPSS for Windows version 20. Descriptive statistics at baseline of age, gender, diagnosis and ethnicity are reported. Fisher's exact test was used with a chi-squared statistic to compare differences in characteristics between the prison discharge and community discharge groups. The prevalence rate ratio (PRR) was also calculated to compare the likelihood of commonly occurring outcomes on discharge between the two samples. Independent samples $t$-tests were conducted to identify any significant differences in mean scores between the prison discharge and community discharge groups on the scale total scores and subscales. Intraclass correlation coefficients (ICCs) were calculated between four raters based on 20 cases to evaluate interrater reliability. The ICCs for all scales and subscales were found to be very good. For the HCR-20 total score the ICC was 0.92, for the SAPROF total it was 0.98, MSRAG total 0.96 and PANSS total 0.93 .

\section{Results}

In the 12-month study period, 794 patients were discharged from 32 MSUs. A quarter $(n=198)$ went to LSUs and $28(4 \%)$ went to HSUs. Nearly three-quarters $(n=568,71.6 \%)$ were discharged to either prison $(n=159,20.1 \%)$ or the community $(n=409$, $51.4 \%$ ) and baseline assessments were conducted on this sample totalling 568. Most were male $(n=516,90.8 \%)$ and over half were White $(n=347,61 \%)$. There were no differences between prison and community groups in terms of gender or ethnicity. Of 409 discharged to a community setting, 178 (43.5\%) were conditionally discharged subject to Section 41 of the Mental Health Act, 2 (0.5\%) were absolutely discharged, 118 (28.9\%) were subject to a Community Treatment Order, and $6(1.5 \%)$ remained on Section 3 of the MHA 1983 (civil order).

Over half of admissions had originally come from prison (53.6\%) and there were significant differences between the two groups based on index offence, referral source, length of stay, age and severity of index offence (see Table 1). The index offence of those discharged to prison was significantly more severe. Nearly $90 \%$ of the prison discharge group were originally referred from prison, compared with $39.4 \%$ of the community discharge group. Those referred from prison were more than twice as likely to be discharged back to prison ( $\mathrm{PRR}=2.25)$ whereas very few of those admitted from the community $(n=3,1.9 \%)$ were eventually discharged to prison.

In terms of psychiatric diagnosis (Table 2), over half the participants had a primary diagnosis of schizophrenia $(n=331$, $58.3 \%)$ and $85(15 \%)$ had a primary diagnosis of personality disorder. Few received a primary diagnosis of substance use disorder (1.1\%), although over two-thirds had a comorbid substance use disorder. There were significantly more patients in the community discharge group with schizophrenia than in the prison discharge group. Nevertheless, a large proportion of the prison discharge group had a primary diagnosis of schizophrenia $(37.7 \%)$ or other psychotic disorder (6.9\%). Individuals discharged to the community were nearly twice as likely to be diagnosed with schizophrenia, whereas those discharged to prison were nearly six times more likely to be diagnosed with a personality disorder. There were no significant differences between the groups based on primary or comorbid diagnoses of substance use disorder (Table 2).

There were significant differences identified between the two groups on all the scales. The prison discharge group scored higher on the HCR-20V3 and MSRAG on measures of risk and symptoms of mental illness; and lower on protective factors measured by the SAPROF (Table 3). The largest difference observed using the HCR-20V3 scales was on the clinical scale, indicating that current 'dynamic' risk was higher among the prison discharge group. The largest difference using the MSRAG was on the risk of future serious offending scale among those discharged to prison.

Overall severity of symptoms of mental illness was significantly higher among the prison discharge group, although mean scores on the PANSS suggested that symptomatology was 


\begin{tabular}{|c|c|c|c|c|c|c|}
\hline Variable & Total & $\begin{array}{l}\text { Community } \\
\text { discharge group }\end{array}$ & $\begin{array}{l}\text { Prison discharge } \\
\text { group }\end{array}$ & $\begin{array}{c}\text { Prison/community } \\
\text { discharge, PRR }(95 \% \mathrm{Cl})\end{array}$ & $\chi^{2}$ & $t$ (d.f.) \\
\hline \multicolumn{7}{|l|}{ Index offence, $n$ (\%) } \\
\hline Violent & 419 (73.8) & $295(72.1)$ & $124(77.5)$ & 1.075 (0.972-1.190) & 1.791 & - \\
\hline Property & $40(7)$ & $21(5.1)$ & 19 (11.9) & $2.296(1.269-4.154)$ & $7.851^{*}$ & - \\
\hline Sexual & $29(5.1)$ & $19(4.6)$ & $10(6.3)$ & $1.336(0.635-2.809)$ & 0.582 & - \\
\hline None & $52(9.2)$ & $50(12.2)$ & $2(1.3)$ & $0.483(0.169-1.386)$ & $16.843^{* * *}$ & - \\
\hline Other & $25(4.4)$ & $21(5.1)$ & $4(2.5)$ & $0.102(0.025-0.412)$ & 1.941 & - \\
\hline Missing & $3(0.5)$ & $3(0.7)$ & $1(0.6)$ & - & - & - \\
\hline \multicolumn{7}{|l|}{ Source of referral, $n$ (\%) } \\
\hline Community & $86(15.1)$ & $83(20.3)$ & $3(1.9)$ & $0.092(0.029-0.286)$ & $30.709 * * *$ & - \\
\hline Prison & 303 (53.3) & $161(39.4)$ & 142 (89.3) & 2.253 (1.976-2.570) & $114.116^{\star * *}$ & - \\
\hline Non-forensic & $40(7.0)$ & $36(8.8)$ & $4(2.5)$ & $0.282(0.102-0.779)$ & $7.084^{\star \star}$ & - \\
\hline High secure & $32(5.6)$ & $29(7.1)$ & $3(1.9)$ & $0.263(0.081-0.850)$ & $5.971^{*}$ & - \\
\hline Medium secure & $62(10.9)$ & $56(13.7)$ & $6(3.8)$ & $0.272(0.120-0.618)$ & $11.868^{* * *}$ & - \\
\hline Low secure & $11(1.9)$ & $10(2.4)$ & $1(0.6)$ & $0.253(0.033-1.967)$ & 2.035 & - \\
\hline Accident and emergency & $3(0.5)$ & $3(0.7)$ & - & - & - & - \\
\hline Other & $28(4.9)$ & $28(6.8)$ & - & - & - & - \\
\hline Missing & $3(0.5)$ & $3(0.7)$ & - & - & - & - \\
\hline \multicolumn{7}{|l|}{ Length of stay, days } \\
\hline Mean (s.d.) & $721.78(896.97)$ & 879.11 (974.5) & $317.08(453.25)$ & - & - & $-6.981^{* * *}(566)$ \\
\hline Median (min-max) & $392(4-7299)$ & $622(7-7299)$ & $143.5(4-3240)$ & - & - & \\
\hline \multicolumn{7}{|l|}{ Age, years } \\
\hline Mean (s.d.) & $36.49(9.95)$ & $37.63(9.74)$ & $33.57(9.91)$ & - & & $-4.060 * * *(566)$ \\
\hline Median (min-max) & $37(18-64)$ & $38(18-64)$ & $32(18-58)$ & - & & \\
\hline Severity of index offence & & & & & - & \\
\hline Mean (s.d.) & $4.21(1.65)$ & $4.07(1.77)$ & $4.58(1.2)$ & - & - & $3.380 * *(563)$ \\
\hline Median (min-max) & $5(0-7)$ & $5(0-6)$ & $5(0-7)$ & - & & \\
\hline
\end{tabular}

mild to moderate whereas the community discharge group were virtually asymptomatic at discharge. The largest difference observed was on the 'Aggression' subscale (measuring level of anger, difficulty in delaying gratification and affective liability) indicating the current risk of violence was significantly higher among the prison discharge group. The largest difference observed with the SAPROF was on the Motivation subscale. Individuals in the prison discharge group were significantly less likely to have protective motivational factors (motivation for treatment and positive attitudes towards authority).

\section{Discussion}

\section{Individuals discharged to prison}

More than one in five patients are now discharged from MSUs back to prison in England and Wales. This is nearly twice as many as observed 15 years ago. The rise in discharges back to prison can partly be explained by the increase in admissions from prison and the greater proportion of prison admissions in the MSU population $(53.6 \%$ from $41 \%)$. This is unsurprising given recent

\begin{tabular}{|c|c|c|c|c|c|}
\hline & \multicolumn{3}{|c|}{$n(\%)$} & \multirow[b]{2}{*}{$\begin{array}{c}\text { Prison/community discharge, } \\
\text { PRR }(95 \% \mathrm{Cl})\end{array}$} & \multirow[b]{2}{*}{$\chi^{2}$} \\
\hline & Total & $\begin{array}{l}\text { Community } \\
\text { discharge group }\end{array}$ & $\begin{array}{l}\text { Prison discharge } \\
\text { group }\end{array}$ & & \\
\hline \multicolumn{6}{|l|}{ Primary diagnosis } \\
\hline Schizophrenia & 331 (58.3) & $271(66.3)$ & $60(37.7)$ & $0.57(0.46-0.70)$ & $38.31 * * *$ \\
\hline Personality disorder & $85(15)$ & $26(6.4)$ & 59 (37.1) & $5.84(3.82-8.91)$ & $85.07 * * *$ \\
\hline Schizoaffective disorder & $59(10.4)$ & $53(13.0)$ & $6(3.8)$ & $0.29(0.13-0.66)$ & $10.37 * *$ \\
\hline Mania/bipolar disorder & $38(6.7)$ & $31(7.6)$ & $7(4.4)$ & $0.58(0.23-1.29)$ & 1.85 \\
\hline Other psychotic disorder ${ }^{a}$ & $21(3.7)$ & $10(2.4)$ & $11(6.9)$ & $2.83(1.23-6.53)$ & $6.04 *$ \\
\hline Depression & $18(3.2)$ & $8(2.0)$ & $10(6.3)$ & $3.22(1.29-8.00)$ & $7.01 *$ \\
\hline Substance use disorder & $6(1.1)$ & $4(1.0)$ & $2(1.3)$ & $1.29(0.24-6.95)$ & 0.09 \\
\hline Anxiety disorder & $3(0.5)$ & $3(0.7)$ & 0 & - & - \\
\hline Intellectual disability & $2(0.4)$ & 0 & $2(1.3)$ & - & - \\
\hline Other diagnosis ${ }^{b}$ & $3(0.5)$ & $3(0.7)$ & 0 & - & - \\
\hline No primary diagnosis & $2(0.4)$ & 0 & $2(1.3)$ & - & - \\
\hline \multicolumn{6}{|l|}{ Comorbid diagnosis } \\
\hline Personality disorder & $154(27.1)$ & $83(20.3)$ & $71(44.7)$ & $2.190(1.69-2.84)$ & $33.98^{* * *}$ \\
\hline Substance use disorder & $377(66.4)$ & 267 (65.3) & $110(69.2)$ & 1.049 (0.93-1.19) & 0.546 \\
\hline Intellectual disability & $28(5)$ & $21(5.1)$ & $7(4.4)$ & $0.845(0.37-1.95)$ & 0.16 \\
\hline
\end{tabular}


Table 3 Comparison of prison and community discharge groups on measures of risk factors, protective factors and mental state

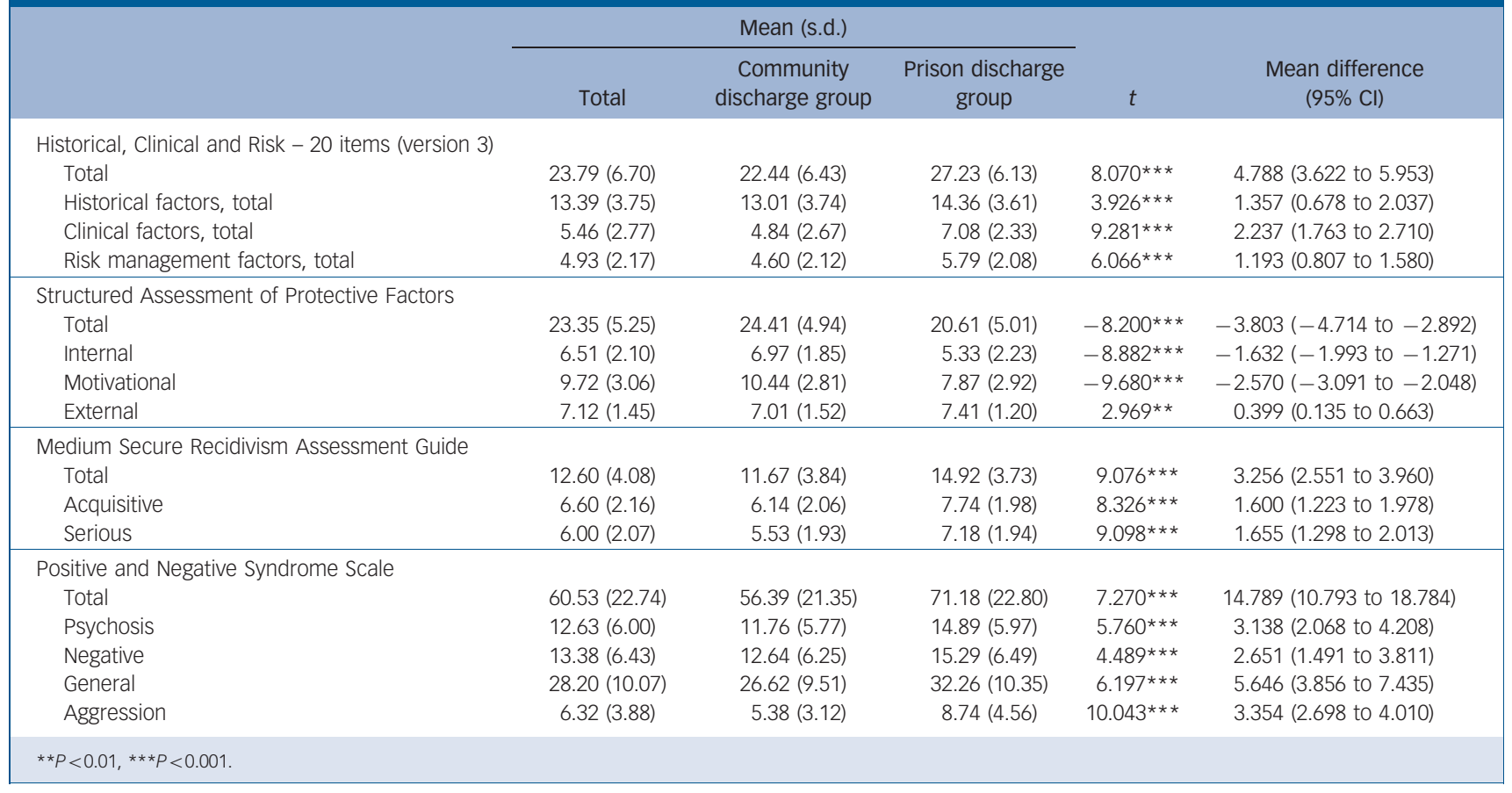

policy initiatives to accelerate transfer from prison to mental health services, ${ }^{17-19}$ although this does not fully account for the relative increase since 1998 . Then, approximately $75 \%$ of prison referrals were discharged via the community compared with about $53 \%$ in this study. This equates to one in four being returned to prison previously and nearly half now. This is very relevant because of the high prevalence of psychiatric morbidity in prison samples, ${ }^{20-22}$ elevated risk identified here in the prison discharge group and probable lack of adequate follow-up on release from prison. $^{23}$

\section{Psychiatric morbidity}

In this study, the community discharge group had a significantly higher rate of schizophrenia, although there remained a high level of psychiatric morbidity in the prison discharge group, with nearly two-thirds having a diagnosis of major mental disorder, nearly half being diagnosed with a serious mental illness and over a third being diagnosed with schizophrenia. This is compounded by the fact that the patients returning to prison were experiencing significantly more psychotic symptoms at the time of discharge compared with the 'asymptomatic' community discharge group. It is not clear from our study why patients were discharged back to prison but it could be that there are now more inappropriate admissions to MSUs since the advent of the Bradley Report, ${ }^{19}$ and the higher rate of personality disorders among the prison discharge group would support this. Those discharged to prison were nearly six times more likely to be diagnosed with a personality disorder and nearly $90 \%$ of the prison discharge group were originally admitted from prison, confirming these as the best indicators of whether a patient would be discharged to prison. It is possible that prison referral and personality disorder diagnosis take priority when clinicians decide the best pathway for prison transfers out of a MSU, especially if this is felt to be a safer option with the advent of prison inreach services. Restrictions on discharge as a result of a Section 49 restriction order might also influence the decision on discharge destination, although this legislation is not new and would not account for the increase in discharges to prison (Section 49 is a restriction order added to a Section 47 transfer direction and discharge has to be approved by the Ministry of Justice). Nevertheless, throughout this pathway there is a real risk that prisoners who are psychotic might not receive the psychiatric services they require. ${ }^{24}$

\section{Risk profile}

The prison discharge group were found to be at a significantly greater risk for future violence and offending. Previous studies have identified that prisoners and those with personality disorder tend to have a more extensive risk history. ${ }^{25}$ They were significantly more likely to be rated as a high risk for violence based on current and dynamic risk factors such as lack of insight, presence of symptoms of mental illness and emotional instability. Combined with the prevailing psychiatric morbidity existing in the prison sample, it is likely most of the risk presented by those discharged to prison would be attributable to deficits in current mental state and functioning. The length of stay of the prison discharge group was significantly less than those discharged to the community, presumably because the prison would normally be seen as a 'safer' and therefore a more expedient discharge option. It is very likely that the criteria for discharge to the community is much more stringent and depends on the availability of suitable post-discharge accommodation and support. Those progressing toward a community pathway would need to prove their stability and progress over a much longer period. It is a concern that those discharged to prison still had significant psychiatric needs and risks, which may not have been addressed in prison prior to release from custody. Despite the growth of inreach services in prison over the past decade, there remains limited evidence to support their effectiveness. ${ }^{26,27}$ Significant concerns have been raised in the recent reviews of inreach services in relation to their establishment and development. Evidence to date suggests that inreach services are inconsistent, lack adequate funding, are understaffed and offer very limited therapeutic interventions. ${ }^{21,28}$ Release from prison is associated with a range of negative outcomes, including increased mortality and suicide, 
and it has been established that very few prisoners with serious mental illness receive mental health services following release and there are serious problems implementing the care programme approach. ${ }^{23,29}$ There is also a fundamental 'legal' limitation within 'non-hospital' prison inreach services, as patients who fail to comply with treatment and subsequently relapse cannot be treated against their will. This is a particular concern when considering that those discharged to prison in this study were significantly more likely to have less motivation to comply with and engage in treatment compared with those discharged to the community.

\section{Limitations}

This study was limited by the fact that patients were not interviewed. However, conducting interviews with patients would require patient consent and it has been found that over 30\% of patients in studies of this type would refuse to consent. ${ }^{8}$ On balance, this would result in an even greater limitation and the absence of patient interviews was compensated by extensive case files that were available for all the individuals who were included and interviews with collaterals who were professional staff currently working with patients, who had a good working knowledge of their history, recent mental state and functioning. Any future studies should consider a mixed-method approach to see if any major differences occur in findings based on interviews with the patient.

The definition of 'community' in this study is not without contention, although it is worth noting that generally there is a distinct lack of clarity and consistency in definitions of community. The community sample was therefore determined to reflect those either in a community placement or in non-secure and/or supported accommodation awaiting more independent living. The type of treatment and intervention received by the sample post-discharge is not reported here. Clearly it would have been of interest to see if patients were linked in with prison inreach services and again post-release to see what type of mental health service follow-up prisoners received, if any at all. This study only included NHS facilities to maintain homogeneity of practice and governance across research sites. Independent sector MSUs were not included but we are confident the findings can be generalised to non-NHS MSUs with the advent of the nationally agreed contract and national standards of practice for MSUs. ${ }^{30}$ Given the growth in such services, ${ }^{20}$ future national studies will need to include independent sector services to enable a more accurate picture of all MSU discharges. It is likely that some of those patients referred to MSUs from other MSUs and HSUs would have originally been referred from prison. This is not accounted for in this study, although we do not believe this would have had a significant impact on the findings regarding discharge to prison.

\section{Policy implications}

The prison discharge group were a higher risk to others, more likely to have a personality disorder, more symptomatic and less motivated to engage in treatment. If more prisoners are being returned to prison with significant needs and risks, then it might be time to consider alternative models of care to reduce the risk to the patient and the public. Reducing transfers to MSU is important, as transfer is expensive and disruptive for the patient, the prison and the MSU. It is far from clear if those returned to prison receive adequate mental health services on return, or appropriate follow-up care on release. ${ }^{31}$

One option might be for MSUs to retain all prison transfers and then discharge them via a mental health pathway, although the costs of an MSU bed per year, at approximately $\mathfrak{E} 176000$ in
2009 and over four times higher than a prison stay per year, ${ }^{31}$ would make this a costly and prohibitive option. The 159 individuals discharged to prison in this sample would have had to stay on average an additional 479 days at the MSU, adding more than $£ 36$ million per year to the MSU bill nationally. This option is also limited by the fact that at least some of the prison transfers would be inappropriate and the individuals probably not amenable to interventions, especially in the case of those with personality disorder.

Another option might be to increase resources to redevelop existing prison inreach teams. There is a wealth of research highlighting the subtherapeutic and even toxic environments in the prison estate ${ }^{32,33}$ indicating that this option might be counterproductive. There is also the problem of not being able to treat those with urgent mental health needs against their will and the probable loss to contact post-release. However, prison mental healthcare can be effective if managed the right way, ${ }^{34}$ where measures are put in place to ensure continuity of care pre- and post-release. ${ }^{35}$ The gate-keeping policies of MSUs are at odds with open access systems operated by inreach teams, where prisoners are often assessed without any prior notice or knowledge. By contrast, patients with comorbid personality disorder may be rejected earlier by MSUs because they are too difficult to manage and fail to comply with MSU admission criteria and the treatment regime.

A new class of prison mental health unit might be indicated, registered with the Care Quality Commission ${ }^{36}$ or attached to an existing registered provider to ensure the quality and effectiveness of care. These would be integrated operationally, but clearly separate to the main prison environment. This would have the benefit of immediate treatment for patients and better continuity of care pre- and post-release. MSUs could then be streamlined to focus on far fewer prison referrals and costs could be reduced by half. Further cost savings would be made by avoiding unnecessary transfers and associated legal bills and the savings made could be redirected to compensate for the redevelopment of new units; recruitment, redeployment and training of staff and improvement in the pathways of care between prison and the community. When considering that nearly half of those transferred from prison to MSUs are returned to prison, then this solution is worthy of consideration by all stakeholders, including policymakers, clinicians, law makers and patient advocates. The benefits would include improved continuity of care pre- and post-release from prison and a reduction in costs and disruption of transfers between prison and MSUs. At the very least, much greater collaborative working between MSUs and prison services is indicated.

\footnotetext{
Michael Doyle, RMN, PhD, Institute of Brain Behaviour and Mental Health, University of Manchester; Jeremy Coid, MB ChB, FRCPsych, MD, Violence Prevention Research Unit, Wolfson Institute of Preventive Medicine, Queen Mary University London; Laura Archer-Power, BSC, Lindsay Dewa, MSC, Alice Hunter-Didrichsen, MSC, Rachel Stevenson, MSC, Verity Wainwright, MSC, Institute of Brain Behaviour and Mental Health, University of Manchester: Costas Kallis, PhD, Simone Ullrich PhD, Violence Prevention Research Unit, Wolfson Institute of Preventive Medicine, Queen Mary University Lon Mary University London; Jenny Shaw, MB ChB, FRCPsych, PhD, Institute of Brain Behaviour and Mental Health, University of Manchester, UK

Correspondence: Michael Doyle, University of Manchester, Jean McFarlane Building, Room 2.311, Oxford Road, Manchester M13 9PL, UK. Email: michael.doyle@manchester.ac.uk

First received 2 Aug 2013, final revision 18 Feb 2014, accepted 14 Mar 2014
}

\section{Acknowledgement}

This article presents independent research commissioned by the National Institute for Health Research (NIHR) under its Programme Grants for Applied Research funding scheme Health Research (NIHR) under its Programme Grants for Applied Research funding scheme
(RP-PG-0407-10500). The views expressed in this article are those of the authors and not necessarily those of the NHS, the NIHR or the Department of Health. 


\section{References}

1 Department of Health and Social Security. Report of the Committee on Mentally Abnormal Offenders (Butler Report). CMMD 624. HMSO, 1975.

2 Sainsbury Centre for Mental Health. Forensic Mental Health Services: Facts and Figures on Current Provision. SCMH, 2007.

3 Ministry of Justice. Statistics of Mentally Disordered Offenders 2008 England and Wales. Ministry of Justice, 2010 (https://www.gov.uk/government/ uploads/system/uploads/attachment_data/file/218001/mentally-disorderedoffenders-2008.pdf).

4 Rutherford M, Duggan S. Forensic mental health services: facts and figures on current provision. Br J Forensic Pract 2008; 10: 4-10.

5 Brown K, Fahy T. Medium secure units: pathways of care and time to discharge over a four-year period in South London. J Forensic Psychiatry Psychol 2009; 20: 268-77.

6 Shah A, Waldron G, Boast N, Coid J, Ullrich S. Factors associated with length of admission at a medium secure forensic psychiatric unit. $J$ Forensic Psychiatry Psychol 2011; 22: 496-512.

7 Maden A, Skapinakis P, Lewis G, Scott F, Burnett R, Jamieson E. Gender differences in reoffending after discharge from medium secure units. Br J Psychiatry 2006; 189: 168-72.

8 Monahan J, Steadman HJ, Silver E, Appelbaum PS, Robbins PC, Mulvey EP, et al. Rethinking Risk Assessment: The MacArthur Study of Mental Disorde and Violence. Oxford University Press, 2001.

9 Doyle M, Dolan M. Predicting community violence from patients discharged from mental health services. Br J Psychiatry 2006; 189: 520-6.

10 Doyle M, Carter S, Shaw J, Dolan M. (2011). Predicting community violence from patients discharged from acute mental health units in England. SOC Psychiatry Psychiatr Epidemiol 2001; 47: 627-37.

11 Webster CD, Douglas KS, Eaves D, Hart SD. HCR 20 - Version 2. Assessing Risk for Violence. Simon Fraser University, 1997.

12 Douglas KS, Hart SD, Webster CD, Belfrage, H. (2013). HCR-20V3: Assessing Risk of Violence - User Guide. Mental Health, Law, and Policy Institute, Simon Fraser University, 2013.

13 De Vogel V, De Ruiter C, Bouman Y, De Vries Robbe M. Structured Assessment of Protective Factors for Violence Risk. Forum Educatief, 2009

14 Hickey N, Yang M, Coid J. (2009). The development of the Medium Security Recidivism Assessment Guide (MSRAG): an actuarial risk prediction instrument. J Forensic Psychiatry Psychol 2009; 20: 202-24.

15 Kay S, Fitszbein A, Opier L. The Positive and Negative Syndrome Scale (PANSS) for schizophrenia. Schizophr Bull 1987; 13: 19-67.

16 Young M, Justice J, Erdberg P. Risk factors for violent behavior among incarcerated male psychiatric patients: a multimethod approach. Assessment 1999; 6: 243-58.

17 Department of Health and HM Prison Service. Changing the Outlook: A Strategy for Developing and Modernising Mental Health Services in Prisons. Department of Health, 2001.
18 Armitage C, Fitzgerald C, Cheong P. Prison in-reach mental health nursing Nurs Stand 2003; 17: 40-2

19 Bradley K. Lord Bradley's Review of People with Mental Health Problems or Learning Disabilities in the Criminal Justice System. Department of Health, 2009.

20 Centre for Mental Health. Pathways to Unlocking Secure Mental Health Care National Mental Health Development Unit, 2011.

21 Shaw J, Senior J, Thornicroft G, Birmingham L, Kendall K, Brooker C. A National Evaluation of Prison Mental Health In-Reach Services. A Report to the National Institute of Health Research. The Offender Health Research Network, 2009 (http://www.ohrn.nhs.uk/resource/Research/Inreach.pdf).

22 Singleton N, Meltzer H, Gatward, R. Psychiatric Morbidity among Prisoners. The Stationery Office, 1998.

23 Lennox C, Senior J, King C, Hassan L, Clayton R, Thornicroft G, et al. The management of released prisoners with severe and enduring mental illness. J Forensic Psychiatry Psychol 2012; 23: 67-75.

24 Coid J, Ullrich S. Prisoners with psychosis in England and Wales: diversion to psychiatric inpatient services? Int J Law Psychiatry 2011; 34: 99-108.

25 McMurran M. Personality Disorder: Expert Paper. National Research and Development Programme on Forensic Mental Health, 2002.

26 Senior J, Birmingham L, Harty M, Hassan L, Hayes A, Kendall K, et al. (2012). Identification and management of prisoners with severe psychiatric illness by specialist mental health services. Psychol Med 2013; 43: 1511-20.

27 Brooker C, Gojkovic, D. The second national survey of mental health in-reach services in prisons. J Forensic Psychiatry Psychol 2009; 20 (suppl 1): $11-28$.

28 Sainsbury Centre for Mental Health. Short Changed: Spending on Prison Mental Health Care. SCMH, 2008.

29 Pyszora NM, Telfer J. Implementation of the care programme approach in prison. Psychiatr Bull 2003; 27: 173-6.

30 NHS England. NHS Standard Contract for Medium and Low Secure Mental Health Services (Adults). NHS England, 2013. 31 Centre for Crime and Justice Studies. Prison and Probation Expenditure, 1999-2009. Centre for Crime and Justice Studies, 2010.

32 Nurse J, Woodcock $P$, Ormsby J. Influence of environmental factors on mental health within prisons: focus group study. BMJ 2003; 327: 480-5.

33 Birmingham L. The mental health of prisoners. Adv Psychiatr Treat 2003; 9: 191-201.

34 Hassan L, Birmingham L, Harty $M$, Jarrett $M$, Jones $P$, King, $C$, et al. Prospective cohort study of mental health during imprisonment. Br J Psychiatry 2011; 198: 37-42.

35 Draine J, Wolff N, Jacoby JE, Hartwell S, Duclos C. Understanding community re-entry of former prisoners with mental illness: a conceptual model to guide new research. Behav Sci Law 2005; 23; 689-707.

36 Care Quality Commission. Application for Registration as a New Service Provider. Care Quality Commission, 2013. 